\title{
ADENOCARCINOMA GÁSTRICO TIPO DIFUSO DE LAUREN: DISSEMINAÇÃO LINFÁTICA EXUBERANTE EM PACIENTE JOVEM
}

\section{Diffuse gastric adenocarcinoma (Lauren classification): exacerbating lymphatic dissemination in young patient}

\author{
Roberto NERY, Thales Paulo BATISTA, Estela K. B. CARVALHO
}

ABCDDV/526

Nery R, Batista TP, Carvalho EKB. Adenocarcinoma gástrico tipo difuso de Lauren: disseminação linfática exuberante em paciente jovem. ABCD Arq Bras Cir Dig 2007;20(1):59-61.

RESUMO - Introdução - O câncer gástrico continua sendo relevante causa oncológica de morte em todo o mundo, com a grande maioria dos casos ocidentais sendo diagnosticados em fase tumoral avançada. O adenocarcinoma representa a quase totalidade dos casos, com pior prognóstico no tipo difuso de Lauren, que acomete mais freqüentemente pacientes jovens. Relato do caso - Agricultor de 20 anos, admitido com plenitude gástrica, dor abdominal e vômitos pós-prandiais, com adenomegalia generalizada de consistência endurecida e indolor, mais evidente, pelo tamanho, na região epigástrica, sub-mandibular e cutânea. Endoscopia digestiva alta demonstrou lesão antral úlcero-infiltrativa com extensas áreas de necrose obstruindo completamente o piloro. Tomografia abdominal mostrou tumoração mal delimitada, estendendo-se ao hipocôndrio direito, epigástrio e comprometendo a segunda porção do duodeno. Estudo anatomopatológico mostrou tratar-se de adenocarcinoma pouco diferenciado com padrão em células de anel de sinete. Foi submetido à gastrojejunostomia paliativa. Conclusão - O adenocarcinoma gástrico pode apresentar-se com comprometimento linfonodal exuberante e sistêmico de grande porte principalmente em baixa idade produzindo adenomegalias volumosas possibilitando somente tratamento paliativo quando diagnosticado.

DESCRITORES - Neoplasias gástricas. Adenocarcinoma.

\section{INTRODUÇÃO}

O câncer gástrico continua sendo importante causa oncológica de morte em todo o mundo ${ }^{1}$, e $80 \%$ dos pacientes diagnosticados no ocidente têm doença avançada ${ }^{2,3}$. Dentre os tumores malignos de estômago, 95\% são adenocarcinomas $^{1}$, classificados histologicamente por Lauren em intestinal e difuso ${ }^{4}$. Em geral, este último é de pior prognóstico ${ }^{5}$ e freqüentemente afeta pacientes mais jovens $s^{2,3,5,6}$.

O objetivo deste relato é notificar a gravidade e extensão que um tumor gástrico pode apresentar em sua fase final, embora que, com este aspecto, ele seja de apresentação rara, especialmente em função da baixa idade que se apresentou.

\section{RELATO DO CASO}

Adulto de 20 anos de idade, agricultor, foi atendido com plenitude gástrica, dor abdominal e vômitos pós-prandiais há 6 meses, precedidos de sintomatologia dispéptica pouco intensa e mal definida. Queixava-se ainda de tumorações submandibular esquerda e epigástrica de surgimento há

Trabalho realizado na Divisão de Cirurgia do Hospital Universitário Lauro Wanderley da Universidade Federal da Paraíba, João Pessoa, PB, Brasil.

Endereço para correspondência: Thales Paulo Batista, e-mail: t.paulo@bol.com.br
2 meses. Apresentava-se emagrecido, com adenomegalia generalizada de consistência endurecida e indolor, mais evidentes pelo tamanho na região epigástrica $(9 \mathrm{~cm})$, submandibular $(3 \mathrm{~cm})$ e transição júgulo-parotídea-masseterina $(4 \mathrm{~cm}$ ) esquerda (Figura 1). Seu abdome era plano, com múltiplas nodulações visíveis e palpáveis superficialmente, difusamente doloroso e com tumoração enrijecida tomando todo o epigástrio.

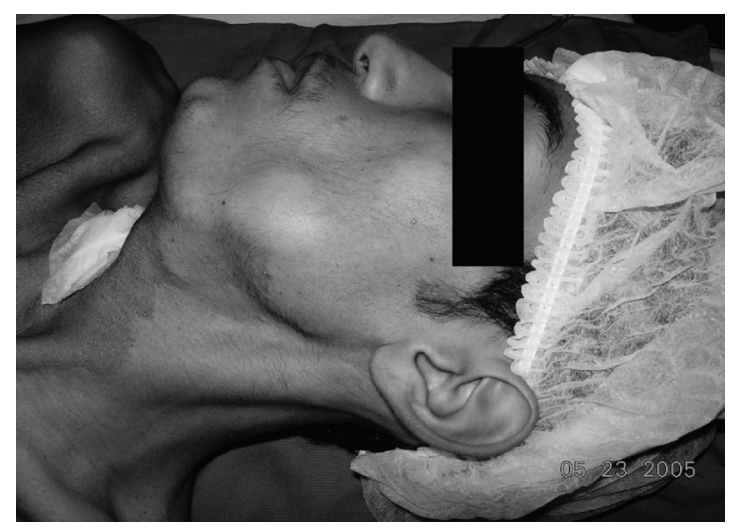

FIGURA 1 - Adenomegalias cérvico-faciais encontradas no paciente 
Foi submetido à endoscopia digestiva alta, que demonstrou lesão antral úlcero-infiltrativa com extensas áreas de necrose obstruindo completamente o piloro, associadas à esofagite grau $\mathrm{C}$ de Los Angeles. A tomografia abdominal mostrou tumoração mal delimitada, extensa, no hipocôndrio direito e epigástrio, comprometendo a segunda porção do duodeno, a vesícula biliar,a região corpo-caudal do pâncreas, o cólon transverso e mesocólon contíguo, além de ascite e acentuada dilatação gástrica (Figura 2). A análise histopatológica de dois linfonodos tóracoabdominais constatou metástases de adenocarcinoma pouco diferenciado com padrão em células de anel de sinete (Estádio IV - T4NxM1).

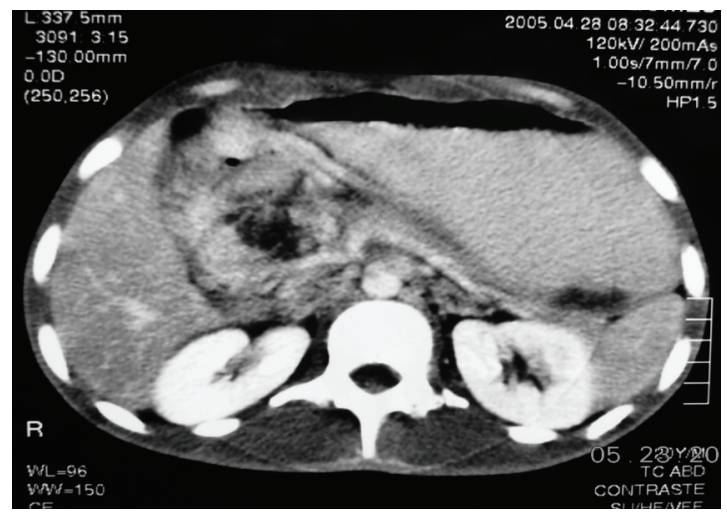

FIGURA 2 - Adenocarcinoma gástrico tipo difuso de Lauren: disseminação linfática exuberante evidenciando a grande extensão tumoral

O paciente foi encaminhado à terapêutica cirúrgica com vistas à paliação tendo-se confirmado no transoperatório a irressecabilidade tumoral. Realizou-se gastrojejunostomia pré-cólica isoperistáltica na face anterior do estômago (Woelfler), tendo em vista às dificuldades técnicas encontradas. Evoluiu sem intercorrências, recebendo alta no $4^{\circ}$ dia de pós-operatório com orientações para o seguimento.

\section{DISCUSSÃO}

Admite-se que o adenocarcinoma gástrico difuso surja espontaneamente a partir da mucosa gástrica nativa, enquanto o tipo intestinal passaria por degeneração maligna após metaplasia intestinal.

O tipo difuso de Lauren apresenta-se macroscopicamente de forma ulcerada ou infiltrativa (linite plástica). Microscopicamente são pouco diferenciados e suas células em anel de sinete a produzem extensa quantidade de mucina e padrão de crescimento infiltrativo. Sua incidência vem se mantendo nos últimos 60 anos nos países ocidentais8. Ainda, acomete sobretudo a cárdia ${ }^{5}$, exibindo disseminação preferencialmente por via transmural e linfática ${ }^{2,3}$, e é mais freqüente nos jovens ${ }^{2,3,5,6}$.

São considerados fatores de risco para o seu surgimento dietas ricas em sal e defumados, tabagismo, grupo sanguíneo $\mathrm{A}$, anemia perniciosa, fatores genéticos, infecção pelo H. pylori e gastrectomia prévia à Billroth II $^{1}$.

O tratamento é essencialmente cirúrgico, levando-se em consideração o estádio e localização do tumor, no entanto, no tipo difuso, a operação de eleição é a gastrectomia to$\mathrm{tal}^{2,3}$. O valor da rádio-quimioterapia ou imunoterapia como adjuvante ao procedimento cirúrgico radical tem sido controvertido ${ }^{9}$, mas, por outro lado, os avanços tecnológicos tem permitido o relato de ressecções videolaparoscópicas oncologicamente adequadas ${ }^{10}$.

O estômago tem sido o órgão do aparelho digestivo mais comumente acometido por câncer entre os jovens ${ }^{11}$, com aumento relativo do número de casos em pacientes abaixo de 41 anos nos útimos 10 anos $^{12}$. Apesar disso, sua ocorrência em pacientes na segunda década de vida é infreqüente, representando de $0,35 \% 12$ a $2,04 \% 13$ dos casos.

Sua morbimortalidade tem sido significativamente correlacionada com a idade dos acometidos, levando alguns autores a considerar abordagem diferencial em função da faixa etária afetada.

O adenocarcinoma mantém-se como o tipo histológico mais freqüente $(91,8 \%)$ entre os pacientes jovens 13 , contudo, devido ao seu comportamento agressivo nesta faixa etária, escassa sintomatologia inicial e diagnóstico tardio, seu prognóstico torna-se muito reservado ${ }^{5}$, com sobrevida mediana de apenas 15 meses $^{4,7}$.

O caso apresentado chama a atenção pela sua disseminação linfática exuberante, produzindo adenomegalias volumosas, grau de agressividade intenso e baixa idade do acometido.

\section{CONCLUSÃo}

O adenocarcinoma gástrico pode apresentar-se com comprometimento linfonodal exuberante e sistêmico de grande porte principalmente em baixa idade, produzindo adenomegalias volumosas e possibilitando somente tratamento paliativo quando diagnosticado. 
Nery R, Batista TP, Carvalho EKB. Diffuse gastric adenocarcinoma (Lauren classification): exacerbating lymphatic dissemination in young patient. ABCD Arq Bras Cir Dig 2007;20(1):59-61.

ABSTRACT - Background - Gastric cancer continues to be a significant and relevant oncologic cause of death in the world, with a great majority of western cases being diagnosed in an advanced tumoral phase. Adenocarcinomas represent the totality of cases having a worst prognosis in Laurens' diffuse type, which is more frequent in young patients. Case report - 20 year-old farmer, admitted with gastric plenitude, abdominal pain, postprandial vomits, with generalized adenomegaly of painless and hardened, becoming more evident due to size in the submandibular and cutaneous epigastric region. Upper digestive endoscopy showed an antral ulcero-infiltrative lesion containing extensive areas of necrosis, which completely obstructed the pyloric region. Abdominal tomography showed an unlimited tumor, extending to the right epigastric hypochondrium, compromising the second portion of the duodenum. Anatomopathologic studies revealed a poorly differentiated adenocarcinoma with signet ring cell pattern. The patient was submitted to palliative gastrojejonostomy. Conclusion - Gastric adenocarcinomas may be present with exacerbating lymphnodal and systemic involvement, especially at young age, generating volumous adenomegalies, turning palliative treatment at the time of diagnosis, the only option available.

HEADINGS - Stomach neoplasms. Adenocarcinoma

\section{REFERÊNCIAS}

1 Filho EDM, Ferraz AAB. Tumores do estômago. In: Ferraz AAB, Ferraz EM, Mathias CAC, ed. Condutas em cirurgia geral. $1^{\text {a }}$ ed. Rio de Janeiro: Medsi; 2003. p. 306-315.

2 Pearlstone DB, Staley CA. Gastric Carcinoma. In: Feig BW, Berger DH, ed The M.D. Anderson surgical oncology handbook. $1^{\text {a }}$ ed. Philadelphia: Lippincott Williams \& Wilkins; 1999. p.139-160.

3 Roukos DH. Current status and future perspectives in gastric cancer management. Cancer treatment reviews 2000; 26:243-255.

4 Lauren F. The two hystopatological main types of gastric carcinomas: diffuse and so called intestinal type carcinoma. Acta Pathol Microbiol Scand 1965; 64:31-49.

5 Rustgi, AK. Neoplasias do estômago. In: Goldman L, Bennett, JC, ed. Cecil - tratado de medicina interna. $21^{\mathrm{a}}$ ed. Rio de Janeiro: Guanabara-Koogan; 2001. p. 818-822.

6 Tomasich FDS, Targa GZ, Alves BRBL, Dias LAN, Kato M. Abordagem diferencial do paciente com câncer gástrico de acordo com a sua idade. Rev Col Bras Cir 2005; 32:99.
7 Crawford JM. Trato gastrintestinal. In: Robbins, SL, ed. Patologia estrutural e funcional. $5^{\text {a }}$ ed. Rio de Janeiro: Guanabara-Koogan; 1996. p. 683-698.

8 Antonioli, DA. Gastric carcinoma and its precursors. Monogr Pathol 1990; 31:144.

9 Goffi FS, Bromberg SH. Gastrectomias subtotais e totais. In: Goffi FS, ed. Técnica cirúrgica - Bases anatômicas, fisiopatológicas e técnicas da cirurgia $4^{\mathrm{a}}$ ed. São Paulo: Atheneu; 2004. p. 568-575.

10 De Paula AL, Macedo AldeV, Shraibman V. Gastrectomia total a D2 videolaparoscópica para o câncer gástrico. Rev Col Bras Cir 2005; 32:13.

11 Ishak G, Assunpção PP, Pereira Jr JL, Macedo NC, Silva MEG. Câncer do aparelho digestivo em pacientes jovens. Rev Col Bras Cir 2005; 32:89.

12 Guimarães Filho W, Dutra LB, Andreollo NA, Lopes LR, Coelho Neto, JS. Câncer gástrico: análise epidemiológica de 1121 casos. Rev Col Bras Cir 2005; 32:379.

13 Guimarães Filho M, Dutra LB, Andreollo NA, Lopes LR. Câncer gástrico em pacientes jovens: estudo de 49 casos. Rev Col Bras Cir 2005; 32:100.

14 Araújo ROC, Sabino FD, Kesley R, Linhares E, Dias JA. Câncer gástrico em pacientes jovens: experiência do INCA. Rev Col Bras Cir 2005; 32:101.

Conflito de interesse: não há Fonte financiadora: não há Recebido para publicação em: 10/08/2006 Aceito para publicação em: 29/11/2006 